\title{
Relación entre la intención de ser activo y la actividad física extraescolar
}

\author{
Juan José Pérez Soto ${ }^{1}$, Eliseo García Cantón ${ }^{2}$, Andrés Rosa Guillamón ${ }^{3}$, Pedro \\ Luís Rodríguez García ${ }^{4}$, Jose Enrique Moral García ${ }^{5}$, Sergio López García ${ }^{6}$ \\ Universidad de Murcia, España ${ }^{1,2,3,4}$ y Universidad de Salamanca, España $a^{5,6}$
}

El objetivo del presente estudio fue relacionar la intención de ser activo y la práctica de actividad física extraescolar en 408 escolares. Para la medición de la actividad física extraescolar se empleó el instrumento de recordatorio de la actividad física extraescolar (PDPAR) y para la intención de ser activo el instrumento de Medición de la Intencionalidad de Ser Activo (MIFA). Los resultados mostraron asociaciones significativas entre la intención de ser activo y las medias de minutos de actividad física extraescolar de moderada a vigorosa intensidad en varones y en mujeres. Se observa como los niveles de actividad física aumentan conforme se incrementa la intencionalidad de ser activo.

Palabras clave: intención, actividad física, escolares, hábitos, salud.

Relationship between intention to be physically active and after-school physical activity The aim of this study was to observe the relationship between the intention to be physically active and the after-school physical activity among 408 schoolchildren. The Previous Day Physical Activity Recall (PDPAR) was administered to measure physical activity, while the Intention to be Physically Active Scale (MIFA) was administered to measure the intention to be physically active. The results showed a significant association between the intention to be active and the average time involved in after-school physical activity from moderate to vigorous intensity in males and females. Physical activity levels increase as the intention to be active increases.

Keywords: intention, physical activity, schoolchildren, habits.

1 Doctor en Educación Física. Profesor asociado de la Universidad de Murcia, Espańa. Dirección postal: Camino Marín, 5, 3E. CP. 30800, Lorca (Murcia).Contacto: juanjose.perez5@ um.es. ORCID: 0000-0003-0248-9885.

2 Doctor en Educación Física, Profesor asociado de la Universidad de Murcia, España. Dirección postal: C/ Doctor Ricardo Cano $142^{\circ} \mathrm{A}$. Contacto: eliseo.garcia@um.es ORCID: 0000-0002-6845-6835.

3 Doctor en Educación Física. Profesor asociado de la Facultad de Educación, Departamento de Educación Física, Universidad de Murcia, Murcia, España. Dirección postal: C/ Doctor Ricardo Cano $142^{\circ} \mathrm{A}$. Contacto: andres.rosa@um.es

4 Doctor en Educación Física, Profesor titular de la Universidad de Murcia, España. Dirección postal: Calle Campus Universitario, 11, 30100 Murcia, España. Contacto: plrodr@um.es

5 Doctor en Educación Física, Universidad de Salamanca, Salamanca, España. Dirección postal: C/ Henry Collet, 52-70, 37007 - Salamanca. Contacto: jemoralga@upsa.es.

6 Doctor en Educación Física por la Universidad de Salamanca, Salamanca, España. Dirección postal: C/ Henry Collet, 52-70, 37007 - Salamanca. Contacto: slopezga@upsa.es 


\section{Relacionamento entre a intençáo de ser ativo e a atividade física fora da escolar}

O objetivo deste estudo foi relacionar a intenção de ser ativo e a prática de atividade física fora da escola em 408 escolares. Para a medição da atividade física fora da escola foi utilizado o instrumento de lembrete de atividade física fora da escola (PDPAR) e para a intenção de ser ativo o instrumento de Medição da Intencionalidade do Ser Ativo (MIFA). Os resultados mostraram associaçôes significativas entre a intenção de ser ativo e os minutos de atividade física fora da escola de intensidade moderada a vigorosa em homens e em mulheres. Observa-se como os níveis de atividade física aumentam à medida que a intencionalidade de ser ativo aumenta.

Palavras-chave: intenção de ser ativo, atividade física, estudantes de escola, hábitos, saúde.

\section{Relation entre l'intention d'être actif et l'activité physique extrascolaire}

L'objectif de la présente étude était d'observer la relation entre l'intention d'être actif et la pratique de l'activité physique extrascolaire chez 408 écoliers. Pour la mesure de l'activité physique extraescolaire a été utilisé l'instrument de rappel de l'activité physique après l'école (PDPAR) et pour l'intention d'être l'instrument de mesure de l'intentionnalité de l'être actif (MIFA). Les résultats ont montré des associations significatives entre l'intention d'être actif et les minutes moyens d'activité physique extraescolaire d'intensité modérée à vigoureuse chez les hommes et chez les femmes. On observe comment les niveaux d'activité physique augmentent à mesure que l'intentionnalité de l'activité physique augmente.

Mots clés: intention, activité physique, école, habitudes, santé. 
Se ha descrito que es en el periodo de ocio extraescolar donde se realiza la mayoría de práctica intencionada de ejercicio físico o deporte por parte de los niños y adolescentes (Arundell, Ridgers, Veitch, Salmon, Hinkley \& Timperio, 2013; Arundell, Fletcher, Salmon, Veitch \& Hinkley, 2016). Dicho periodo transcurre durante la tarde, cuando los escolares han acabado las actividades académicas. Sin embargo, son escasos los escolares que se adhieren con continuidad a actividades físicas extraescolares una vez entrados en la adolescencia (Middelbeek \& Breda, 2013).

En las encuestas de hábitos deportivos de la población española (MEC, 2015), se observaba que tan solo un $38 \%$ de los sujetos ubicados en edad adolescente practicaba deporte todos los días, habiendo un $19 \%$ de los mismos que no practicaba ningún día a la semana. Es por ello, que la intervención para el fomento de práctica de actividad física se debería ubicar en edades previas a la adolescencia (Boreham \& Riddoch, 2001; Shephard \& Trudeau, 2000; Van Der Horst, Paw, Twisk \& Van Mechelen, 2007).

Algunas de las teorías que intentan explicar desde un punto de vista psicoconductual la realización de actividad física incluyen la intención, la motivación de emprender el comportamiento, así como la actitud previa de los sujetos (Bandura, 2004; Noar \& Zimmerman, 2005). La intención de ser activo se ha mostrado como uno de los predictores más destacados de la actividad física en estudios realizados con niños y adolescentes (Araújo-Soares, McIntyre \& Sniehotta, 2009; Chatzisarantis, Biddle \& Meek, 1997; Kamtsios, 2011; Roberts, Maddison, Magnusson \& Prapavessis, 2010; Sallis, Prochaska \& Taylor, 2000). Sin embargo, suele ser una variable que se analiza de forma asociada a otros determinantes de la actividad física, tales como las habilidades de planificación (Cao, Schüz, Xie \& Lippke, 2013), la autoeficacia, planificación de acción y afrontamiento (Araújo-Soares, McIntyre 
\& Sniehotta, 2009) o el autoconcepto físico (Moreno, Moreno \& Cervelló, 2007).

En el contexto español, Castillo, Balaguer y Tomás (1997) hallaron que en chicos, las variables que más predicen la práctica de ejercicio físico intenso fueron la autovaloración de la aptitud deportiva y la intención de ser activo en el futuro. En las chicas, la intención de ser activo predecía el ejercicio físico intenso y la práctica deportiva general. En otro estudio con escolares españoles y que utilizó el test de intención de ser activo se afirma que los promedios de intención de ser activo eran altos. Sin embargo, dichos datos no fueron contrastados con su práctica habitual de actividad física (Arias, Castejón \& Yuste, 2013).

Asimismo, en una investigación realizada con profesores, Blázques Manzano, León-Mejía y Molina (2015) hallaron que aquellos que tenían más intención realizaban más actividad física. Sin embargo, dicha asociación se encontraba relacionada con el tiempo de ocio que disponían los participantes y otras variables sociales. Por el contrario, en un estudio de revisión, Rhodes y de Bruijn (2013) hallaron que tan solo un $54 \%$ de los adultos que tienen intención, practican actividad física. En base a lo anterior, la asociación entre las variables parece no cumplirse en todos los casos en adultos. En otra investigación con la población adulta, hallaron que la influencia familiar era el principal determinante de práctica de actividad física (Ruiz-Juan, Pieron \& Baena Extremera, 2012). Por ello, es preciso destacar que la asociación entre intención y práctica de actividad física, se suele observar de forma más clara en edades más tempranas de acuerdo con la literatura científica.

Sobre la base de lo expuesto y teniendo en cuenta la escasez de estudios que se centran en los hábitos de los escolares en el tiempo extraescolar, el objetivo del presente estudio fue observar la relación entre la intención de ser activo y la práctica de actividad física extraescolar de los sujetos en edad escolar. 


\section{Método}

\section{Participantes}

Para la realización del estudio, se escogió un total de 408 escolares espańoles, 203 varones (49\%) y 205 mujeres (51\%) cursando Educación Primaria en municipios del sureste español (muestra de conveniencia). Las edades estaban comprendidas entre los 10 y 12 años (alumnos de $5^{\circ}$ y $6^{\circ}$ curso). El estudio fue de tipo relacional, descriptivo y de carácter transversal.

Una vez obtenido el consentimiento informado para participar en el estudio por parte del equipo directivo de los centros, padres y participantes; se llevó a cabo la investigación siguiendo las normas deontológicas reconocidas por la Declaración de Helsinki (2008) y siguiendo las recomendaciones de Buena Práctica Clínica de la CEE (1990).

\section{Medición}

Para la medición de la intencionalidad de ser físicamente activo, se empleó el cuestionario Medida de la Intencionalidad para ser Físicamente Activo (MIFA), creado por Hein, Müür y Koka (2004) y adaptado al contexto español por Moreno, Moreno y Cervello (2007) con una muestra de adolescentes españoles. Además, es un instrumento que ha sido recientemente empleado con escolares de educación primaria españoles (Arias, Castejón \& Yuste, 2013), en un estudio donde se analizaron sus propiedades psicométricas mostrando una alta consistencia interna $(\alpha=.80)$ y fiabilidad temporal $(I C C=.79)$. Se trata de un cuestionario ampliamente utilizado en escolares que consta de diversas afirmaciones, y que deben ser respondidas haciendo uso de una escala tipo Likert que oscila desde 1 (nada) a 4 (mucho).

En cuanto a la medición de la actividad física extraescolar, se empleó el Previous Day Physical Activity Recall (PDPAR). Dicho instrumento fue validado por Weston, Petosa y Pate (1997) con adolescentes estadounidenses y posteriormente para escolares de quinto y sexto grado 
por Trost, Ward, McGraw y Pate (1999). La versión utilizada para el presente estudio fue la adaptación transcultural para alumnado en contexto hispanohablante realizada por Rodríguez, Pérez-Soto, GarcíaCantó y Rosa (2014).

El instrumento consta de 19 periodos de 30 minutos que van desde las 14:00h hasta las 23:00h. En ese tiempo el sujeto debe recordar la principal actividad que realizó el día anterior. En la primera sesión, se exponía mediante el instrumento de aplicación y apoyados en una presentación digital, cómo se debía completar el cuestionario. Posteriormente, lo relatado por los sujetos se equiparó a intensidades MET con la planilla actividad-intensidad MET (Trost, Ward, McGraw \& Pate, 1999) y se clasificó en tres niveles: minutos de actividades de intensidad moderada (3-6 MET), minutos de actividades de intensidad vigorosa ( $>6 \mathrm{MET}$ ) y el sumatorio de minutos de actividades de moderada a vigorosa intensidad (> $3 \mathrm{MET}$ ). En la presente investigación, la actividad física se obtuvo mediante la suma de minutos totales en la que los sujetos se mantenían realizando actividades físicas de moderada a vigorosa intensidad (> $3 \mathrm{MET}$ ). La calificación de los sujetos se obtuvo a través del promedio de MET de los tres días que se realizó medición. Dichos MET, derivaban de las actividades de los participantes que se situaban en una intensidad por encima de 3 MET.

\section{Procedimiento}

Se estableció un protocolo de actuación dentro de los centros escolares de tres días seguidos semanales. El primer día, previo a la realización del cuestionario de actividad física, se implementó el cuestionario de medición de intencionalidad de ser activo MIFA. Durante esos tres días, el alumnado tenía que responder a un cuestionario de actividad física (PDPAR) cada día.

Los exploradores siguieron un procedimiento de entrenamiento que consistía en plantear la finalidad del estudio y realizar una puesta en común. Tras ello, se aplicaba el cuestionario y se recogían datos para 
analizar la fiabilidad de las pruebas. Finalmente se determinaba la fiabilidad intra e interexplorador del mismo.

Tras el proceso de entrenamiento, se procedió a realizar el estudio. El cuestionario de medición de la actividad física (PDPAR) fue objeto de una explicación en la primera sesión, durante el resto de sesiones lo realizaban de forma autónoma.

\section{Análisis de datos}

Los datos fueron analizados con el programa estadístico SPSS (v.15.0). Se realizó el Test de Kolmogorov-Smirnov para observar la distribución normal de la variable, obteniendo $p>0.05$. Tras ello, se hizo uso de la estadística inferencial mediante análisis de varianza simples (one-way ANOVA) en ambos sexos, con el objeto de analizar las diferencias entre grupos. En los análisis se relacionaban las categorías obtenidas en el MIFA sobre la intencionalidad de ser activo (nada, poco, bastante y mucho), con el nivel de actividad física (promedio de minutos envueltos en AFMV). Este último actuaba como variable dependiente y continua dentro de la investigación. Asimismo, en el caso de las variables continuas se calcularon medias y desviaciones típicas, y para las variables categóricas, se utilizaron porcentajes. Del mismo modo, se realizó un análisis confirmatorio post hoc para confirmar las relaciones encontradas por el análisis de varianza.

\section{Resultados}

En la Tabla 1 se pueden apreciar los sujetos encuadrados en las cuatro opciones de respuesta en función de las medias obtenidas en la escala de Medida de la Intencionalidad de Ser Activo (MIFA). La prueba de chi cuadrado ha detectado diferencias significativas entre sexos en la intencionalidad de ser activo $\left(c^{2}=33,118 ; p<.01\right)$ de los sujetos analizados. Un $21 \%$ del total de la muestra afirma tener poca 
intención de ser activo, mientras la mayoría de sujetos (59.6\%) promedian bastante intención de ser activos. Las mujeres superan a los hombres en las categorías de nada ( $90 \%$ vs $9 \%$ ) o poca ( $73 \%$ vs $26 \%$ ) intención de ser activo, mientras que los hombres son mayoría en las categorías de bastante $(57.2 \%$ vs $42.8 \%$ ) y mucha ( $58.8 \%$ vs $41.2 \%$ ) intencionalidad de ser activo.

\section{Tabla 1}

Medida de la intencionalidad de ser físicamente activo (MIFA) por sexo

\begin{tabular}{|c|c|c|c|c|c|}
\hline & & & \multicolumn{2}{|c|}{ Sexo } & \multirow{2}{*}{ Total } \\
\hline & & & Varón & Mujer & \\
\hline \multirow[t]{16}{*}{ MIFA } & \multirow[t]{4}{*}{ Nada } & Recuento & 1 & 10 & 11 \\
\hline & & $\%$ de MIFA & $9.1 \%$ & $90.9 \%$ & $100.0 \%$ \\
\hline & & $\%$ del total & $.2 \%$ & $2.5 \%$ & $2.7 \%$ \\
\hline & & Residuos corregidos & -2.7 & 2.7 & \\
\hline & \multirow[t]{4}{*}{ Poco } & Recuento & 23 & 63 & 86 \\
\hline & & $\%$ de MIFA & $26.7 \%$ & $73.3 \%$ & $100.0 \%$ \\
\hline & & $\%$ del total & $5.6 \%$ & $15.4 \%$ & $21.1 \%$ \\
\hline & & Residuos corregidos & -4.8 & 4.8 & \\
\hline & \multirow[t]{4}{*}{ Bastante } & Recuento & 139 & 104 & 243 \\
\hline & & $\%$ de MIFA & $57.2 \%$ & $42.8 \%$ & $100.0 \%$ \\
\hline & & $\%$ del total & $34.1 \%$ & $25.5 \%$ & $59.6 \%$ \\
\hline & & Residuos corregidos & 3.7 & -3.7 & \\
\hline & \multirow[t]{4}{*}{ Mucho } & Recuento & 40 & 28 & 68 \\
\hline & & $\%$ de MIFA & $58.8 \%$ & $41.2 \%$ & $100.0 \%$ \\
\hline & & $\%$ del total & $9.8 \%$ & $6.9 \%$ & $16.7 \%$ \\
\hline & & Residuos corregidos & 1.6 & -1.6 & \\
\hline \multirow{3}{*}{\multicolumn{2}{|c|}{ Total }} & Recuento & 203 & 205 & 408 \\
\hline & & $\%$ de MIFA & $49.8 \%$ & $50.2 \%$ & $100.0 \%$ \\
\hline & & $\%$ del total & $49.8 \%$ & $50.2 \%$ & $100.0 \%$ \\
\hline \multicolumn{3}{|c|}{$\mathrm{c}^{2}=33.118 ; p<0.005$} & & & \\
\hline
\end{tabular}

MIFA: medida de intencionalidad de ser físicamente activo.

Nota: Nada: promedio de 1 a 1.99 puntos; Poco: promedio de 2 a 2.99 puntos; Bastante: promedio de 3 a 3.99 puntos; Mucho: promedio de 4 puntos. 
En la Tabla 2 se muestran los resultados del cuestionario de intención de ser activo en varones muestran asociaciones significativas con las medias de minutos de actividad física extraescolar de moderada a vigorosa intensidad de los escolares $(p=.01)$. De ese modo, aquellos que obtuvieron unas medias de actividad física superiores se encuadraban en los niveles medio $(M=100.26 \pm 45.94)$ y alto $(M=94.54 \pm 48.27) \mathrm{de}$ intencionalidad de ser activo.

\section{Tabla 2}

Intencionalidad de ser fisicamente activo y su relación con los minutos de actividad fisica en varones

\begin{tabular}{lccccc}
\hline $\begin{array}{l}\text { Intencionalidad de ser } \\
\text { activo en varones }\end{array}$ & $N$ & $M$ & $D E$ & $F$ & $p$ \\
\hline Bajo & 88 & 69.31 & 48.20 & & \\
Medio & 38 & 100.26 & 45.94 & & \\
Alto & 77 & 94.54 & 48.27 & & \\
Total & 203 & 84.67 & 49.49 & 8.19 & .005 \\
\hline
\end{tabular}

Nota: Bajo: de 1.80 a 3.39 puntos; Medio: de 3.40 a 3.79 puntos; Alto: de 3.80 a 4 puntos; Media: media de minutos empleados en actividad física extraescolar de moderada a vigorosa intensidad.

Por el contrario, los sujetos con una intención baja de ser activo arrojan las medias más bajas de actividad física $(M=69.31 \pm 48.20)$. El análisis post hoc (Tabla 3), detectó asociaciones entre el nivel bajo con el medio $(p=.003)$ y el nivel bajo con alto $(p=.003)$.

En mujeres (Tabla 4), se observan asociaciones significativas en los 3 niveles de intencionalidad de ser físicamente activo respecto a las medias de minutos de actividad física de los participantes. Se observa como los niveles de actividad física van aumentando conforme sube la intencionalidad de ser activo $(p<.001)$. 


\section{Tabla 3}

Análisis post hoc de la relación entre la intencionalidad de ser físicamente activo y los minutos de actividad física en varones

\begin{tabular}{|c|c|c|c|c|c|c|}
\hline \multirow[t]{2}{*}{$\begin{array}{l}\text { (I) Intencio- } \\
\text { nalidad de } \\
\text { ser activo en } \\
\text { varones }\end{array}$} & \multirow[t]{2}{*}{$\begin{array}{l}(\mathrm{J}) \text { Intencio- } \\
\text { nalidad de } \\
\text { ser activo en } \\
\text { varones }\end{array}$} & \multirow{2}{*}{$\begin{array}{c}\begin{array}{c}\text { Diferencia } \\
\text { de medias } \\
(\mathrm{I}-\mathrm{J})\end{array} \\
\begin{array}{c}\text { Límite } \\
\text { inferior }\end{array}\end{array}$} & \multirow{2}{*}{$\begin{array}{c}\begin{array}{c}\text { Error } \\
\text { típico }\end{array} \\
\text { Límite } \\
\text { superior }\end{array}$} & \multirow{2}{*}{$\begin{array}{c}\text { Sig. } \\
\text { Límite } \\
\text { inferior }\end{array}$} & \multicolumn{2}{|c|}{$\begin{array}{c}\text { Intervalo de } \\
\text { confianza al } 95 \%\end{array}$} \\
\hline & & & & & $\begin{array}{l}\text { Límite } \\
\text { superior }\end{array}$ & $\begin{array}{l}\text { Límite } \\
\text { inferior }\end{array}$ \\
\hline \multirow[t]{2}{*}{ Bajo } & Medio & $-30.94\left(^{*}\right)$ & 9.28 & .003 & -53.35 & -8.53 \\
\hline & Alto & $-25.22\left(^{*}\right)$ & 7.46 & .003 & -43.24 & -7.21 \\
\hline \multirow[t]{2}{*}{ Medio } & Bajo & $30.94\left(^{*}\right)$ & 9.28 & .003 & 8.53 & 53.35 \\
\hline & Alto & 5.71 & 9.48 & 1.000 & -17.17 & 28.60 \\
\hline \multirow[t]{2}{*}{ Alto } & Bajo & $25.22\left(^{*}\right)$ & 7.46 & .003 & 7.21 & 43.24 \\
\hline & Medio & -5.71 & 9.48 & 1.000 & -28.60 & 17.17 \\
\hline
\end{tabular}

* La diferencia de medias es significativa al nivel .05 .

\section{Tabla 4}

Intencionalidad de ser fisicamente activo y su relación con los minutos de actividad fisica en mujeres

\begin{tabular}{lccccc}
\hline $\begin{array}{l}\text { Intencionalidad de ser } \\
\text { activo en mujeres }\end{array}$ & $N$ & $M$ & $D E$ & F & $p$ \\
\hline Bajo & 73 & 40.95 & 32.58 & & \\
Medio & 65 & 64.00 & 35.78 & & \\
Alto & 67 & 88.65 & 46.41 & & \\
Total & 205 & 63.85 & 43.16 & 26.709 & .000 \\
\hline
\end{tabular}

Nota: Bajo: de 1 a 2.79 puntos; Medio: de 2.80 a 3.39 puntos; Alto: de 3.40 a 4 puntos; Media: media de minutos empleados en actividad física extraescolar de moderada a vigorosa intensidad.

El análisis post hoc (Tabla 5) encontró relaciones entre los niveles bajo y medio $(p=.002)$, bajo y alto $(p<.001)$, y medio y alto $(p=.001)$ de intencionalidad de ser físicamente activo. 


\section{Tabla 5}

Análisis post hoc de la relación entre la intencionalidad de ser físicamente activo y los minutos de actividad física en mujeres

\begin{tabular}{|c|c|c|c|c|c|c|}
\hline \multirow[t]{2}{*}{$\begin{array}{l}\text { (I) Intencio- } \\
\text { nalidad de } \\
\text { ser activo en } \\
\text { mujeres }\end{array}$} & \multirow[t]{2}{*}{$\begin{array}{l}(\mathrm{J}) \text { Intencio- } \\
\text { nalidad de } \\
\text { ser activo en } \\
\text { mujeres }\end{array}$} & \multirow{2}{*}{$\begin{array}{c}\begin{array}{c}\text { Diferencia } \\
\text { de medias } \\
(\mathrm{I}-\mathrm{J})\end{array} \\
\begin{array}{c}\text { Límite } \\
\text { inferior }\end{array}\end{array}$} & \multirow{2}{*}{$\begin{array}{c}\begin{array}{c}\text { Error } \\
\text { típico }\end{array} \\
\text { Límite } \\
\text { superior }\end{array}$} & \multirow{2}{*}{$\begin{array}{c}\text { Sig. } \\
\text { Límite } \\
\text { inferior }\end{array}$} & \multicolumn{2}{|c|}{$\begin{array}{c}\text { Intervalo de } \\
\text { confianza al } 95 \%\end{array}$} \\
\hline & & & & & $\begin{array}{l}\text { Límite } \\
\text { superior }\end{array}$ & $\begin{array}{l}\text { Límite } \\
\text { inferior }\end{array}$ \\
\hline \multirow[t]{2}{*}{ Bajo } & Medio & $-23.04\left(^{*}\right)$ & 6.57 & .002 & -38.92 & -7.16 \\
\hline & Alto & $-47.69\left(^{*}\right)$ & 6.52 & .000 & -63.45 & -31.94 \\
\hline \multirow[t]{2}{*}{ Medio } & Bajo & $23.04\left(^{*}\right)$ & 6.57 & .002 & 7.16 & 38.92 \\
\hline & Alto & $-24.65\left(^{*}\right)$ & 6.71 & .001 & -40.86 & -8.44 \\
\hline \multirow[t]{2}{*}{ Alto } & Bajo & $47.69\left(^{*}\right)$ & 6.52 & .000 & 31.94 & 63.45 \\
\hline & Medio & $24.65\left(^{*}\right)$ & 6.71 & .001 & 8.44 & 40.86 \\
\hline
\end{tabular}

Nota: ${ }^{*}$ La diferencia de medias es significativa al nivel .05 .

\section{Discusión}

La intencionalidad de ser activo en jóvenes ha sido estudiada como un factor determinante para la realización de práctica físico-deportiva real en el periodo extraescolar (Bandura, 2004). En el análisis de nuestra muestra, se observa que el $88 \%$ de los varones, el $65 \%$ de las mujeres y el 76,3\% del total, afirman tener bastante o mucha intención de ser activo. Estos resultados son diferentes por categorías de respuesta y sexos, siendo los varones los que superan a las mujeres en la categoría de bastante $(57.2 \%$ vs $42.8 \%$ ) y de mucha intención de ser activo (58.8\% vs $41.2 \%)$. Las mujeres quedarían por encima en las categorías de nada y poca intención de ser activo. Estos resultados van en la línea de lo encontrado en investigaciones similares, así Castillo, Balaguer y Tomás (1997) con escolares del sureste español hallaron que el 91\% de los varones y el $80 \%$ de las mujeres afirmaba tener intención de ser 
activo en el futuro. Ruiz-Pérez, Ramón-Otero, Palomo-Nieto, RuizAmengual y Navia-Manzano (2014), también con jóvenes españoles de entre 11 y 16 años, señalaban que el $87 \%$ de la muestra realizaría o probablemente realizaría actividad física a la edad de 20 años. Moreno, Moreno y Cervelló (2007) encontraron diferencias significativas por sexo empleando el mismo instrumento de intencionalidad que en el presente estudio, siendo los varones los que puntuaban más alto en todas las dimensiones.

Un estudio con 657 escolares españoles de Castilla La-Mancha, Murcia y Valencia, indicaba que la intención futura de práctica deportiva tenía una tendencia alta con un valor medio de 3.94 sobre 5 puntos. Los autores también encontraron diferencias por edades, siendo la intencionalidad de práctica futura de los participantes de 11 a 12 años mayor que la de los del grupo de entre 9 y 10 años (Arias, Castejón \& Yuste, 2013).

En nuestra investigación, cuando se relacionó la intención de ser activo con la actividad física, se encontraron asociaciones significativas tanto en varones como en mujeres. Aquellos varones que quedaban encuadrados en baja intencionalidad de ser activo, fueron los que arrojaban medias de actividad física inferiores. En cuanto a las mujeres, se observa como la media de minutos de actividad física realizada va creciendo conforme se encuadran en las tres categorías (baja, media, alta), siendo la alta intencionalidad de ser activo la que se asocia con más minutos de actividad física.

Los estudios que correlacionan la intencionalidad de ser activo con la actividad física en la literatura son escasos. Los que lo hacen, suelen abarcar distintas variables predictoras y calcular el valor predictor que tienen la práctica físico-deportiva en la intención de ser activo. Por lo general todos encuentran relaciones significativas entre las dos variables. En esa línea, en un estudio con escolares portugueses la intención fue el factor más determinante entre la autoeficacia, la planificación y el afrontamiento (Araújo-Soares, McIntyre \& Sniehotta, 2009). En otro estudio con adolescentes neozelandeses, la actividad física subjetiva medida mediante cuestionario se relacionó más fuertemente con 
la intencionalidad activo. Sin embargo, dentro del mismo estudio la actividad física medida mediante podómetro se relacionó de forma más significativa con la eficacia en la tarea (Roberts, Maddison, Magnusson \& Prapavessis, 2010). En otro estudio con escolares entre 11 y 15 años, se demostró que las intenciones predecían la actividad física cuando los participantes experimentaban las decisiones de forma autónoma (Chatzisarantis, Biddle \& Meek, 1997).

En un estudio realizado en Grecia con una muestra de 573 varones y 620 mujeres de entre 11 y 18 ańos, se analizaron las actitudes e intenciones hacia el ejercicio, la percepción subjetiva del esfuerzo y disfrute, las autopercepciones y los niveles de actividad física. Los resultados revelaron que los participantes con actitudes más positivas e intención hacia el ejercicio participaban más en actividad física moderada y vigorosa (Kamtsios, 2011).

Bajo la importancia de desarrollar una intencionalidad de ser activo en los escolares para incidir en sus niveles de actividad física, Ruiz-Pérez, Ramón-Otero, Palomo-Nieto, Ruiz-Amengual y NaviaManzano (2014), realizaron un estudio para evaluar los factores predictores de la intención de ser activo con 480 escolares españoles de 11 a 16 años. Las variables que mejor predecían la intención de practicar actividad físico-deportiva en el futuro fueron las autopercepciones de competencia deportiva, de forma física y de salud, la práctica de las personas más significativas y la pertenencia activa a un club deportivo. Asimismo, también ha sido descrito que el clima social y los modelos de educación deportiva desde el área de Educación Física puede ser predictores de la intencionalidad de ser activo y la práctica de actividad física extraescolar (Martínez, Méndez-Giménez y Valverde, 2016).

El presente estudio no está exento de limitaciones, en primer lugar, se utilizó un cuestionario de autoinforme para evaluar los hábitos de actividad física de los escolares, habiendo sido descrito que puede dar lugar a sobrevalorar los niveles de actividad física en algunas ocasiones. Sin embargo, se buscaba la practicidad y versatilidad para el ámbito de escuela primaria; aportando información relevante sobre los distintos tipos de actividades en los que participaban los sujetos (sedentarias, 
moderadas y vigorosas). Además, el hecho de que el recordatorio sea de la tarde anterior, facilita el recuerdo y acota la posible limitación de la falta de memoria, ya que otros instrumentos instan a recordar la actividad física de los últimos siete días. Por otro lado, los resultados deben interpretarse con cautela, al no poderse establecer una relación causal en las relaciones encontradas debido a la naturaleza transversal del estudio y no haberse controlado otras posibles variables influyentes, tales como el desarrollo psicomotor (Garaigordobil, 1999) o la competencia percibida (Babic et al., 2014).

Por tanto, el presente estudio corrobora que la relación entre la intencionalidad de ser activo y la actividad física habitual autoinformada en escolares de etapa primaria del sureste español. Futuros estudios longitudinales deberán evaluar si dicha asociación permanece en el tiempo, sobre todo cuando se adentren en la adolescencia. Los docentes de Educación Física, deben de desarrollar programaciones y metodologías atractivas para los escolares, de forma que tengan intención de ser activos en su periodo de ocio extraescolar. Especial atención requieren las primeras etapas de la adolescencia.

\section{Referencias}

Araújo-Soares, V., McIntyre, T. \& Sniehotta, F. F. (2009). Predicting changes in physical activity among adolescents: the role of selfefficacy, intention, action planning and coping planning. Health Educ Res, 24(1), 128-39. https://doi.org/10.1093/her/cyn005

Arias, J. L., Castejón, F. J. \& Yuste, J. L. (2013). Propiedades psicométricas de la escala de intencionalidad de ser físicamente activo en Educación Primaria. Revista de Educación, 362, 485-505. https://doi.org/10.4438/1988-592X-RE-2013-362-239.

Arundell, L., Fletcher, E., Salmon, J., Veitch, J. \& Hinkley, T. (2016). A systematic review of the prevalence of sedentary behavior during the after-school period among children aged 5-18 years. International Journal of Behavioral Nutrition and Physical Activity, 13, 93. https://doi.org/10.1186/s12966-016-0419-1 
Arundell, L., Ridgers, N. D., Veitch, J., Salmon, J., Hinkley, T. \& Timperio, A. (2013). 5-year changes in afterschool physical activity and sedentary behavior. Am J Prev Med, 44(6), 605-611. https://doi.org/10.1016/j.amepre.2013.01.029

Babic, M. J., Morgan, P. J., Plonikoff, R. C., Lonsdale, C., White, R. L. \& Lubans, D. R. (2014). Physical activity and physical self-concept in youth: systematic review and meta-analysis. Sports Med, 44(11), 1589-601. https://doi.org/10.1007/s40279-014-0229-z

Bandura, A. (2004). Health promotion by social cognitive means. Health Education Behavior, 31, 143-164. https://doi. org/10.1177/1090198104263660

Blázquez Manzano, A., León-Mejía, A. \& Feu Molina, S. (2015). Intención y práctica de actividad física en maestros españoles. Cuadernos de Psicología del Deporte, 15(2), 163-170. https://doi. org/10.4321/S1578-84232015000200018

Boreham, C. \& Riddoch, C. (2001). The physical activity, fitness and health of children. J Sports Sci., 19, 915-929. https://doi. org/10.1080/026404101317108426

Cao, D., Schüz, N., Xie, G. \& Lippke, S. (2013). Planning skills moderate the intention-planning cognitions-behaviour relation: a longitudinal study on physical activity in Chinese adolescents. Res Sports Med, 21(1), 12-23. https://doi.org/10.1080/1543862 7.2012.738441

Castillo, I., Balaguer, I. \& Tomás, I. (1999). Predictores de la práctica de actividades físicas en niños y adolescentes. Anales de Psicología, 13(2), 189-200.

Chatzisarantis, N.L.D., Biddle, S. J.H. \& Meek, G.A. (1997). A self-determination theory approach to the study of intentions and the intention-behaviour relationship in children's physical activity. British Journal of Health Psychology, 2, 343-360. https:// doi.org/10.1111/j.2044-8287.1997.tb00548.x.

Garaigordobil, M. (1999) Evaluación del desarrollo psicomotor y sus relaciones con la inteligencia verbal y no verbal. Revista Iberoamericana de Diagnóstico y Evaluación Psicológica, 8(2), 9-36. 
Hein, V., Muur, M. y Koka, A. (2004). Intention to be physically active after school gradutation and its relationship to three types of intrinsic motivation. European Physical Education Review, 10, 5-19. https://doi.org/10.1177/1356336X04040618.

Kamtsios S. (2011). Differences in attitudes towards exercise, perceived athletic ability, perceived physical attractiveness and participation in physical activity in children and adolescents aged 10 to 18 years old. Journal of Sport and Health Research, 3(3), 129-142.

Martínez, D., Méndez-Giménez, A. \& Valverde, J. J. (2016). Efectos del modelo Educación Deportiva en el clima social del aula, la competencia percibida y la intención de ser físicamente activo: un estudio prolongado en primaria. SPORTTK: Revista iberoamericana de ciencias del deporte, 5(2), 153-166.

Middelbeek, L. \& Breda, J. (2013). Obesity and Sedentarism: Reviewing the Current Situation Within the WHO European Region. Current Obesity Reports, 2(1), 42-49. https://doi.org/ 10.1007/s13679-013-0054-y

Ministerio de Educación, Cultura y Deporte (2015). Encuesta de hábitos deportivos 2015. Madrid: Secretaría General Técnica.

Moreno, J. A., Moreno, R. \& Cervelló, E. (2007). El autoconcepto físico como predictor de la intención de ser físicamente activo. Psicología y Salud, 17(2), 261-267.

Noar, S. M. \& Zimmerman, R.S. (2005). Health behavior theory and cumulative knowledge regarding health behaviors: Are we moving in the right direction? Health Education Research, 20, 275-290. https://doi.org/10.1093/her/cyg113

Rhodes, R. \& de Bruijn, G. (2013). How big is the physical activity intention-behaviour gap? A meta-analysis using the action control framework. British Journal of Health Psychology, 18, 296309. https://doi.org/10.1111/bjhp.12032

Roberts, V., Maddison, R., Magnusson, J. \& Prapavessis, H. (2010). Adolescent physical activity: does implementation intention have a role? J Phys Act Health, 7(4), 497-507. https://doi.org/ 10.1111/bjhp. 12032 
Rodríguez-García, P. L., Pérez-Soto, J.J., García-Cantó, E. \& Rosa-Guillamón, A. (2015). Cross-cultural adaptation of a questionnaire that evaluates physical activity among children aged 10 and 11. Arch argent pediatr, 113(3), 198-204. https:// doi.org/10.5546/aap.2015.eng.198

Ruiz-Juan, F., Pieron, M. \& Baena Extremera, A. (2012). Socialización de la actividad físico-deportiva en adultos: relación con familia, pareja y amigos. Revista Iberoamericana de Diagnóstico y Evaluación Psicológica, 34(1), 34-59.

Ruiz-Pérez, L.M., Ramón-Otero, I., Palomo-Nieto, M., Ruiz-Amengual, A. \& Navia-Manzano, J. A. (2014). La Intención de Practicar en el Futuro en Escolares Adolescentes. Kronos, 13(2).

Sallis, J. F., Prochaska, J.J. \& Taylor, W. C. (2000). A review of correlates of physical activity of children and adolescents. Medicine and Science in Sports and Exercise, 32, 963-975. https:// doi.org/10.1097/00005768-200005000-00014

Shephard, R. \& Trudeau, F. (2000). The legacy of physical education: influences on adult lifestyle. Pediatric Exercise Science, 12, 34-50. https://doi.org/10.1177/1356336X020081005.

Trost, S. G., Ward, D. S., McGraw, B. \& Pate, R. (1999). Validity of the Previous Day Physical Activity Recall (PDPAR) in Fifth-Grade Children. Pediatric Exercise Science, 11(4), 341-348. https://doi. org/10.1123/pes.11.4.341

Van Der Horst, K., Paw, M.J., Twisk, J. W \& Van Mechelen, W. (2007). A brief review on correlates of physical activity and sedentariness in youth. Med Sci Sports Exerc., 39(8), 1241-1250. https://doi. org/10.1123/pes.11.4.341.

Weston, A.T., Petosa, R. \& Pate, R. (1997). Validation of an instrument for measurement of physical activity in youth. Med Sci Sports Exerc, 29(1), 138-143.https://doi.org/10.1097/00005768-19970100000020

Recibido: 2 de enero, 2018 Revisado: 19 de setiembre, 2018 Aceptado: 15 de enero, 2019 